\title{
Mesenteric panniculitis a case report and review of literature
}

\author{
DM SHAH, SB PATEL, SR SHAH, KG GOSWAMI
}

Ind J Radiol Imag 2005 15:2:191-192

Key words : - Mesenteric panniculitis, CT

\section{INTRODUCTION:}

Mesenteric panniculitis is a benign disease characterized by nonspecific inflammation and fibrosis involving the adipose tissue of the mesentery. Mesenteric panniculitis is an uncommon disease of unknown etiology. We present a case of mesenteric panniculitis showing classical CT features.

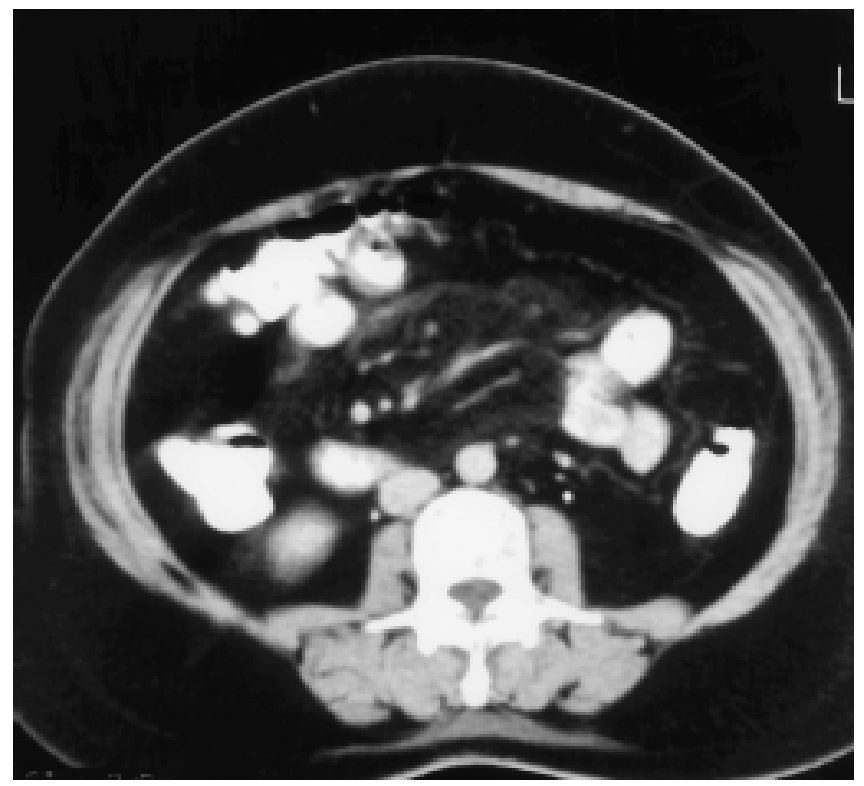

Fig 1

\section{CASE REPORT:}

A 54 years female patient presented with complains of pain in epigastric and umbilical region. On clinical examination there is soft tissue fullness in umbilical region. There is no evidence of guarding or rigidity. Laboratory tests were unremarkable. We performed CT scan of abdomen and pelvis on Hitachi CTW 2000 scanner. Oral and intravenous contrast was given and 10 $\mathrm{mm}$ serial sections were taken from domes of diaphragm upto pelvis. CT scan showed presence of a well defined, partially encapsulated mass at the root of mesentery. The density of the mass is slightly higher than that of the surrounding adipose tissue with a ground glass appearance. The mass encases mesenteric vessels. Small bowel loops were displaced peripherally. Rest of the examination was unremarkable. We diagnosed it as Mesenteric Panniculitis. Patient did not undergo biopsy or surgery so histopathological confirmation could not be obtained but imaging features were typical of Mesenteric Panniculitis.

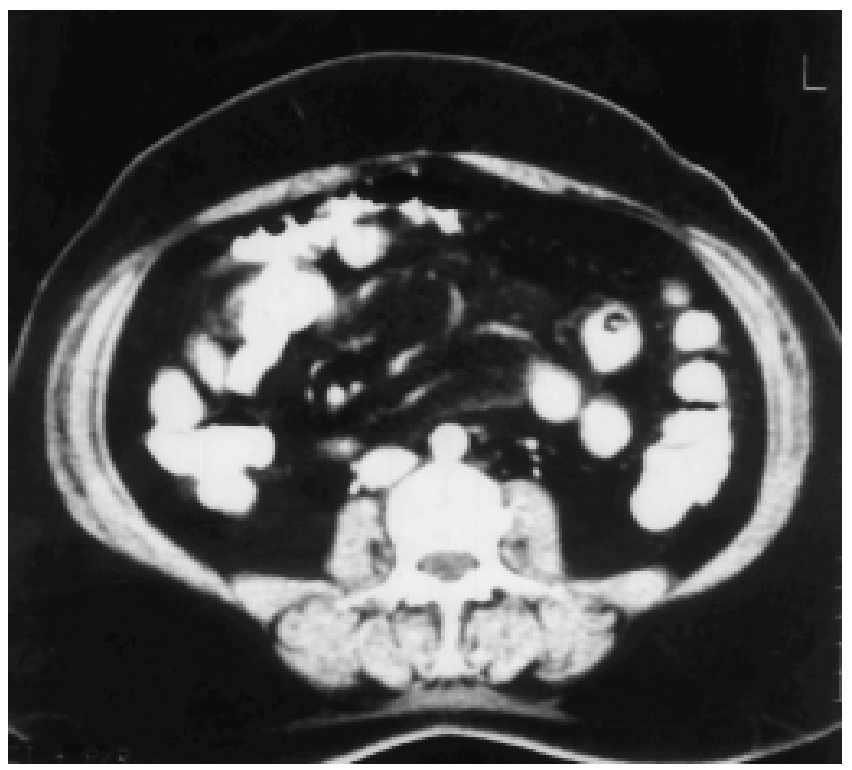

Fig 2

\section{DISCUSSION:}

Mesenteric panniculitis is a rare disease of unknown pathogenesis that involves the fat tissue of small bowel mesentery or less commonly of the colonic mesentery. It occurs more frequently in late adult life. Either sex may be affected although there is a higher incidence in males (1.8: 1) [1]. Common presenting symptoms include

From the Department of Radio-diagnosis, Gujarat Cancer and Research Institute, Asarwa, AHMEDABAD - 380016

Request for Reprints: Dr. Dipal M. Shah, Junior Lecturer, Department of Radio-diagnosis, Gujarat Cancer and Research Institute, Asarwa, AHMEDABAD - 380016 
abdominal pain, fever, nausea, vomiting, diarrhea or constipation and rectal bleeding. A poorly defined abdominal mass is frequently palpated and may occasionally discovered incidentally in asymptomatic patients. The disease has a generally benign but erratic course.

Two types of inflammatory disease of the mesentery have been described [2]. When the predominating histological component is inflammatory or fatty, the process is known as mesenteric panniculitis, mesenteric lipogranuloma, isolated lipodystrophy of mesentery or pseudotumoral intraabdominal panniculitis [2]. If the fibrosis is the predominating component, the disease is known as retractile mesenteritis, subperitoneal multifocal sclerosis or chronic fibrous mesenteritis [2]. The term mesenteric panniculitis should be used in all cases with acute or subacute inflammatory process of mesentery, and retractile mesenteritis for those cases with a chronic source complicated by fibrosis and retraction.

Macroscopic findings show enlarged mesentery. Mesenteric involvement may be diffuse nodular or multinodular. Microscopic findings include anomalous fatty cells with foamy cytoplasm and infiltrations by monocytes, lymphocytes, lipid-laden macrophages and foreign body giant cells. There are areas of fatty necrosis and a variable degree of fibrosis. The fatty necrosis may calcify [2].

Plain radiography provides very few findings. Sonography may show a well-defined hyperechoic mass with small, frequently central hypoechoic areas, an irregularly shaped hypoechoic area or a heterogenous but predominantly hyperechoic mass [1]. Colour Doppler shows mesenteric vessels within the mass and high resistance flow in jejunal and ileal branches [5].

Computed Tomography correlates well with surgical findings. There is presence of encapsulated, heterogenous masses localized to the root of mesentery or adjacent to the intestinal loops [2]. Thickening of the intestinal wall is occasionally seen. The CT image is varied, depending on the stage of the disease and whether the inflammatory, fatty or fibrous component is predominant. When diffuse inflammation of mesenteric fat is the predominant feature, the mass appears as a well-defined mainly fatty tumor with diffuse serpeginous soft tissue densities throughout the root of the small bowel mesentery. The soft tissue densities represent inflammatory infiltrates and fibrotic areas together with the mesenteric vessels. Mesenteric vessels are displaced and surrounded by the involved fat but not invaded [2]. Neither benign nor malignant neoplasm surrounds the mesenteric vessels in this way without causing vessel invasion and/or displacement. When fibrosis is predominant component, the CT image is similar to previous description, appearing as a mass of predominantly soft tissue density. Small calcifications are frequently identified by pathologists but are rarely recognized on plain film or CT studies, however, there are only two previous descriptions in which calcifications were radiologically visible [4].

Mesenteric panniculitis must be distinguished from several other disorders such as necrotising pancreatitis, mesenteric lymphoma, carcinoid, leiomyosarcoma, lipomas, liposarcoma, myelolipoma, angiomyolipoma, teratoma, peritoneal carcinomataosis, abscess and hematomas [5].

\section{REFERENCES:}

1. Orlando Catalano, Bianca Cusati: Sonographic detection of mesenteric panniculitis: case report and literature review. J. Clin Ultrasound 25: 141-144,1997.

2. J Mata, L Inaraja, J Martin, A Olazabal and M Castilla: CT features of mesenteric panniculitis. JCAT vol.11, No.6: 1021-1023, 1987.

3. Katz ME, Heiken JP, Glazer HS, Lee JKT. Intraabdominal panniculitis: clinical, radiographic and CT features. AJR 1985; 145:293-296.

4. Perez-Fontan FJ, Soler R, Sanchez J, er al: Retractile mesenteritis involving the colon: barium enema, sonographic and CT findings. AJR 147: 937-940, 1986.

5. Bellin MF, Thi Huong Du Le, Sarfaty C, et al: MRI and colour Doppler in sclerosing mesenteritis. Eur Radiol 2: 373-376,1992. 